\title{
Complete Teager Energy Operator: A Novel Method for Enhancing Micro-impact Signal
}

\section{Xu Dong}

Huanggang Normal University

huipeng li ( $\nabla$ yeshuip@163.com )

Wuhan University of Science and Technology https://orcid.org/0000-0002-7830-849X

\section{Research}

Keywords: Large-scale rotating machinery, Micro shock pulse, Incipient fault, Complete Teager energy operator, Enchancment

Posted Date: September 7th, 2021

DOI: https://doi.org/10.21203/rs.3.rs-835244/v1

License: (c) (1) This work is licensed under a Creative Commons Attribution 4.0 International License. Read Full License 


\title{
Complete Teager energy operator: A novel method for enhancing micro-impact signal
}

\author{
Xu Dong ${ }^{1}$, Huipeng $\mathrm{Li}^{2,3}$ \\ 1 School of Architectural Science and Engineering, Huanggang Normal University, Huanggang, Hubei, 438000, \\ PR China \\ 2 School of Physics and Electronic Information, Huanggang Normal University, Huanggang, Hubei, 438000, PR \\ China \\ 3 Engineering Research Center for Metallurgical Automation and Measurement Technology of Ministry of \\ Education, Wuhan University of Science and Technology, Wuhan, Hubei 430081, PR China
}

Abstract: The output of conventional Teager energy operator (TEO) is approximately equal to the square product of the instantaneous amplitude and the instantaneous frequency $\left(A^{2} \Omega^{2}\right)$. The original TEO can effectively enhance the transient shock components and suppress the non-impacting elements, and it also changes the frequency distribution of the original shock. In this paper, a complete Teager energy operator is proposed, and its expression is more exact than original method. By keeping the positive and negative distribution of the shock signal $x(t)$, the fundamental frequency energy of the impulses can be effectively enhanced. The incipient fault characteristics of large-scale rotating machinery are typically micro shock pulse, extremely weak and mixed with heavy noise. Preprocessing the fault signal and enhancing the micro shock component are essential means to extract the early fault features. In the experiment part, the applicability of the proposed method is verified by the simulated micro impact signal, the common bearing fault data-sets and the practical measured data of the test bench.

Keyords: Large-scale rotating machinery, Micro shock pulse, Incipient fault, Complete Teager energy operator, Enchancment.

\section{Introduction}

In the undamped free vibration system, the general solution is $A \cdot \cos (\omega \cdot t+\varphi)$, thus, the output of conventional Teager energy operator is $T_{\text {energy }}=A^{2} \Omega^{2}$, which is an approximation of $A^{2} \cdot \sin ^{2} \Omega$ [1]. When large rotating machinery is working at low speed and heavy load, once bearing pitting or defect is developed, it will produce periodic impact signal due to the impact between parts. Through the analysis of the collected vibration signal, it is found that the impulse caused by the fault presents the sine exponential attenuation property [2]. That is to say, the function characteristic of shock signal satisfies the general solution form of damped free vibration system [3].Base on that, this paper proposes an influence factor $\gamma$, termed $T_{\text {energy }}=\gamma \cdot A^{2} \cdot \Omega^{2}$, which provides the exact output form of Teager energy operator. In addition, the output value of conventional Teager energy operator is nonnegative. But in practice, the collected fault signals of large, low-speed and heavy-duty machinery are bipolar. Teager energy operator can enhance the instantaneous change of micro impulses in fault signal, however, its output is consistently positive, which changes the amplitude and frequency distribution of the micro shock signal. Therefore, by distinguishing the polarity of the original signal, the output of Teager energy operator is $( \pm) \gamma \cdot A^{2} \cdot \Omega^{2}$, that is consistent with the positive and negative characteristics 
of the raw signal, At the same time, the frequency distribution of original micro shock signal is maintained. This kind of Teager energy operator is named as complete Teager energy operator (CTEO) in this paper. It can effectively enhance the magnitude of transient impact components and maintain the frequency distribution of the signal.

With the development of industrial technology, engineering mechanical equipment is developing towards the tendency of maximization, complexity and automation. Under complex working conditions, the transmission part of equipment is easily damaged. Its early defect may cause unexpected equipment accidents, and even endanger personal safety [4]. Therefore, mechanical equipment condition monitoring and fault diagnosis technology have attracted the attention of the industry. Taking the case of the mechanical equipment running under low speed and heavy load, the early shock fault often reflects the characteristics of low signal-to-noise ratio (SNR) and long period. Affected by strong background noise and attenuation of signal transmission process, the fault characteristics are extremely sparse and weak, and it is difficult to extract fault features directly. Therefore, it is necessary to preprocess the original fault signal through denoising and enhancing useful features to improve the SNR. The most commonly used denoising methods are wavelet [5-6], empirical mode decomposition [7], sparse decomposition [8], etc. In addition, Teager energy operator is often applied to enhance the impulse components in fault signal [9]. Teager energy operator is an energy model of dynamics, which is introduced into the field of signal processing. It is a parameter free method, simple and effective [10], and has high real-time performance. The wavelet method needs to select the appropriate basis function and related parameters in advance [11], its validity for the bearing early fault of large rotating machinery with low-speed needs to be further verified by experiments. Therefore, Teager energy operator is often used to improve the SNR of such fault signals [12].

The remainder of the paper is organized as follows. Section 2 introduces the theoretical principles of Teager energy operator and describes the proposed Teager energy operator framework. In Section 3, the proposed framework is verified by simulation analysis. In Section 4,public experiment data set analysis and engineering data analysis are conducted. Finally, conclusions are presented in Section 5.

\section{The proposed method}

In present section, we will introduce a novel identification method of shock signal enhancement factor in detail. Firstly, the model of vibration system is introduced and analyzed. After that, the framework of TEO is deduced. Then, a complete enhancement factor is proposed.

\subsection{Framework of single-freedom vibration system}

A single degree of freedom vibration system usually consists of a mass $m$ of directional vibration, an elastic element $k$ connected between the vibration mass and the foundation and a damping $c$ in motion. The system is shown in Fig.1. According to Newton's second law, the motion differential equation of vibration system can be established as:

$$
m \ddot{x}+c \dot{x}+k x=0
$$

where $x$ is the position of the vibration mass $m, c$ is the damping coefficient, $k$ denotes the elastic coefficient. $\dot{x}$ and $\ddot{x}$ represent the first and second derivatives of $x$, respectively. Eq. (1) can be analyzed in two cases:

(1) Eq. (1) is a single degree of freedom undamped free vibration when $c=0$, and its general solution is as follow: 


$$
x=A\left(\sin \omega_{n} t+\varphi_{n}\right)
$$

where $A$ is the amplitude, $\varphi_{n}$ is the arbitrary phase, $\omega_{n}=\sqrt{\frac{k}{m}}$ is the natural frequency of the vibration system.

(2) When $c \neq 0$, Eq. (1) presents a single degree of freedom mass-spring-damper system. The expression of general solution is deduced as:

$$
x=A e^{-\zeta t} \sin \left(\omega_{r} t+\varphi_{r}\right)
$$

where $A$ is the amplitude and $\varphi_{r}$ is the initial phase. $\zeta=\frac{c}{2 m}$ is the attenuation coefficient, and the higher value indicates greater damping and faster amplitude attenuation. $\omega_{r}=\sqrt{\omega_{n}^{2}-\zeta^{2}}$ is the angular frequency of the reduced motion attenuation.

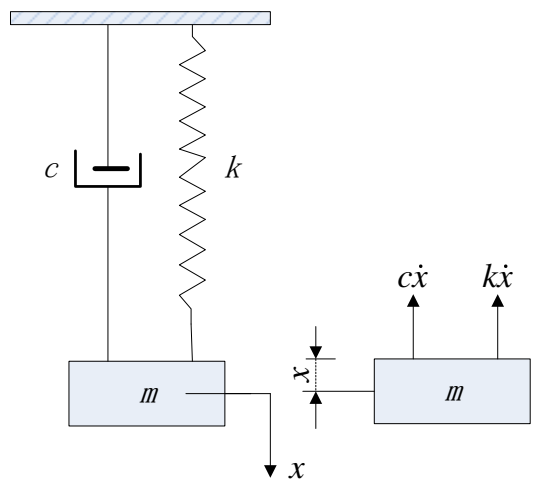

Fig.1. Single degree of freedom vibration system.

\subsection{Teager energy operator}

For continuous signals $x(t)$, a kind of nonlinear energy tracking difference operator, named Teager energy operator(TEO)[13] is used to track and capture the instantaneous change of narrowband signals. By simple mathematical analysis, the nonlinear energy tracing operator for continuous signal is defined as Eq. (4), referred to as $\psi_{c}$ :

$$
\psi_{c}[x(t)]=\left(\frac{d x(t)}{d t}\right)^{2}-x(t) \frac{d^{2} x(t)}{d t^{2}}=[\dot{x}(t)]^{2}-x(t) \ddot{x}(t)
$$

where $\dot{x}(t)$ and $\ddot{x}(t)$ denote the first and second derivatives of the signal $x(t)$ versus time $t$, respectively.

The function form of shock signal excitated by large rotating machinery early fault conforms to the general solution form of damped free vibration system. As shown in Fig.1, For discrete signals $x(n)$, Kaiser [14] proposed the differential Teager energy operator, which can quickly track the energy changes of the signal with three close samples. The discrete TEO is defined as Eq. (5):

$$
\psi_{c}[x(n)]=[x(n)]^{2}-x(n-1) x(n+1)
$$

According to Eq. (3), it can be concluded:

$$
\left\{\begin{array}{l}
x_{n-1}=A \cdot e^{-\zeta(n-1)} \cdot \sin (\Omega \cdot(n-1)+\varphi) \\
x_{n}=A \cdot e^{-\zeta n} \cdot \sin (\Omega \cdot n+\varphi) \\
x_{n+1}=A \cdot e^{-\zeta(n+1)} \cdot \sin (\Omega \cdot(n+1)+\varphi)
\end{array}\right.
$$


In Eq. (6), $A$ is the amplitude, $\varphi$ is the initial phase, $\Omega=\frac{\omega}{2 \pi}$ is the frequency, and $\zeta$ denotes attenuation coefficient. Apparently,

$$
\begin{aligned}
x_{n+1} \cdot x_{n-1}= & {\left[A \cdot e^{-\zeta(n+1)} \cdot \sin (\Omega \cdot(\mathrm{n}+1)+\varphi)\right] \cdot\left[A \cdot e^{-\zeta(n-1)} \cdot \sin (\Omega \cdot(\mathrm{n}-1)+\varphi)\right] } \\
& =A^{2} \cdot e^{-2 \zeta n} \cdot[\sin (\Omega \cdot(n+1)+\varphi) \cdot \sin (\Omega \cdot(n-1)+\varphi)]
\end{aligned}
$$

According to the formula

$$
\begin{gathered}
\sin (a+b) \cdot \sin (a-b)=\sin ^{2} a-\sin ^{2} b, \text { we can get } \\
x_{n+1} \cdot x_{n-1}=A^{2} \cdot e^{-2 \zeta n} \cdot\left(\sin ^{2}(\Omega \cdot n+\varphi)-\sin ^{2} \Omega\right) \\
=x_{n}^{2}-A^{2} \cdot e^{-2 \zeta n} \cdot \sin ^{2} \Omega .
\end{gathered}
$$

Hence,

$$
x_{n}^{2}-x_{n+1} \cdot x_{n-1}=e^{-2 \zeta n} \cdot A^{2} \cdot \sin ^{2} \Omega .
$$

$\sin \Omega$ is approximately equal to $\Omega$ for small values of $\Omega$, that is, $\sin \Omega \approx \Omega$. When $\zeta=0$, the expression of Teager energy operator is obtained as follows:

$$
T=x_{n}^{2}-x_{n+1} \cdot x_{n-1} \approx A^{2} \cdot \Omega^{2}
$$

where $T$ is an approximation of Teager energy operator output, which is approximately equal to the square product of the instantaneous amplitude and the instantaneous frequency of a vibration signal. Due to the high frequency variation of transient impact, this method can effectively enhance the transient impact components.

\subsection{The proposed CTEO model}

In this paper, a complete factor $\gamma$ is introduced and the Eq. (7) is modified as follows:

$$
T=x_{n}^{2}-x_{n+1} \cdot x_{n-1}=e^{-2 \zeta n} \cdot A^{2} \cdot \sin ^{2} \Omega=A^{2} \cdot \Omega^{2} \cdot \frac{\sin ^{2} \Omega}{\Omega^{2}} \cdot e^{-2 \zeta n}
$$

Let $\gamma=\frac{\sin ^{2} \Omega}{\Omega^{2}} \cdot e^{-2 \zeta n}$, Eq. (9) can be expressed as:

$$
T=x_{n}^{2}-x_{n+1} \cdot x_{n-1}=e^{-2 \zeta n} \cdot A^{2} \cdot \sin ^{2} \Omega=\gamma \cdot A^{2} \cdot \Omega^{2}
$$

In order to keep the output of Teager energy operator as the product of instantaneous amplitude and instantaneous frequency square, the above formula is modified as follows:

$$
T=\frac{T}{\gamma}=A^{2} \cdot \Omega^{2}
$$

where $T$ is the complete Teager energy operator proposed in this paper, which is the exact output of $T$. Notably, $\gamma=\frac{\sin ^{2} \Omega}{\Omega^{2}} \cdot e^{-2 \zeta n} \in(0,1)$, so the output of energy operator $T$ is greater than that of $T$. That is, $T$ is an enhanced expression of $T$.

The instantaneous angular frequency $\Omega$ is irrelevant to the attenuation coefficient $\zeta$ and the 
initial amplitude $A$ of the signal. Considering calculation simplicity, we assume $e^{-\zeta n}=1$ and $A=1$, and Eq. (7) becomes

$x_{n}^{2}-x_{n+1} \cdot x_{n-1}=\sin ^{2} \Omega$.

Thus, $\sin \Omega= \pm \sqrt{x_{n}^{2}-x_{n+1} \cdot x_{n-1}}$.

It can be concluded that:

$\Omega=\arcsin \pm \sqrt{x_{n}^{2}-x_{n+1} \cdot x_{n-1}}$

So, $\frac{\sin ^{2} \Omega}{\Omega^{2}}=\operatorname{Sa}^{2}(\Omega)=$

$\begin{cases}1, & \text { for } \Omega=0 \\ \operatorname{Sa}^{2} \arcsin \pm \sqrt{x_{n}^{2}-x_{n+1} \cdot x_{n-1}} & , \text { for } \Omega \neq 0 \text { and }\left(x_{n}^{2}-x_{n+1} \cdot x_{n-1}>0\right) \operatorname{and}\left(\sqrt{x_{n}^{2}-x_{n+1} \cdot x_{n-1}}<1\right) \\ \frac{\left(z^{2}+z \sqrt{z^{2}-1}-1\right)^{2}}{\left(z+\sqrt{z^{2}-1}\right)^{2} \ln ^{2}\left|z+\sqrt{z^{2}-1}\right|}, & \text { for }|\mathrm{z}|=\left|\sqrt{x_{n}^{2}-x_{n+1} \cdot x_{n-1}}\right| \text { and }\left|x_{n}^{2}-x_{n+1} \cdot x_{n-1}\right|>1 .\end{cases}$

According to Section 2.2, Teager energy operator can enhance the energy of micro impulses in fault signal. However, its output is consistently positive, which changes the amplitude and frequency distribution of the micro shock signal. Therefore, in order to keep the characteristic of the original signal, the demodulated signal of the complete Teager energy operator is $( \pm) \gamma \cdot A^{2} \cdot \Omega^{2}$ by prejudging the positive and negative property of $x_{n}[\cdot]$, and the representation signal is consistent with the symbol of the $x_{n}[\cdot]$. Thus, the frequency distribution of the original signal is maintained.

\section{Simulation and validation}

When the bearing of large-scale mechanical equipment has pitting corrosion or defect fault, periodic impact signal will be generated due to the impact between parts [15]. By analyzing the vibration signal captured by sensor, it can be found that the shock signal caused by the fault presents exponential attenuation sine property. In order to verify the validity of the proposed complete TEO method, in this section, a typical simulated fault excitation signal of rolling bearing is employed for analysis. The simulated signal $x(t)$ is composed of periodic impulse signal $s(t)$ and additive white Gaussian noise $n(t)$.

$$
\left\{\begin{array}{l}
x(t)=s(t)+n(t) \\
s(t)=\sum_{i} A_{i} h\left(t-i T-\tau_{i}\right) \\
h(t)=e^{-C t} \sin \left(2 \pi f_{n} t\right) \\
A_{i}=1+A_{0} \sin \left(2 \pi f_{r} t\right)
\end{array}\right.
$$

where $A_{0}$ is the initial amplitude of the impulse signal and $A_{i}$ is the amplitude of the impulse sequence. $T$ is the cyclic period, $\tau_{i}$ is the random tiny slippage during each $T$, usually considered as $0.01 T-0.02 T . C$ denotes the damping coefficient. $f_{r}$ is the rotation frequency of the simulated transmission shaft and $f_{n}$ is the resonance frequency. The bearing fault signal can be simulated by setting appropriate parameter values. Here, the simulated signal parameters are set as follow: $C=750$, 
$f_{r}=1 \mathrm{~Hz}, f_{n}=3000 \mathrm{~Hz}, A_{0}=0.5$. In addition, the characteristic frequency of simulation fault signal is $f_{\text {inner }}=1 / T=100 \mathrm{~Hz}$, the signal-to-noise ratio ( $\mathrm{SNR}$ ) is $-11 \mathrm{~dB}$, the sampling frequency $f_{s}$ is set to $12 \mathrm{kHz}$ and the collected data length $\mathrm{L}=12000$. The simulation fault signal is shown in Fig. 2 .

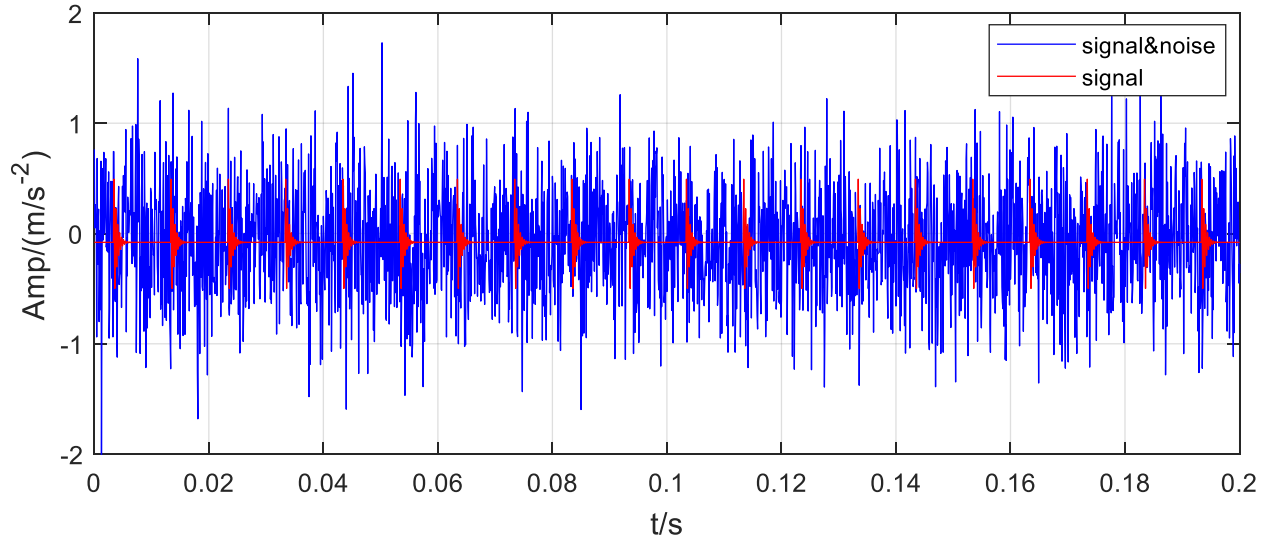

Fig.2. The simulation of the shock signal

In this test, as shown in Fig.2, the red waveform is pure periodic impulse signal, and the blue one is impact signal mixed with strong white noise. The envelope spectrums of the above signals are shown in Fig. 3(a) and (b), respectively. From the Hilbert envelope spectrum in Fig.3 (a), the fault characteristic frequency $f_{i}(100 \mathrm{~Hz})$ and its harmonics $(200 \mathrm{~Hz}, 300 \mathrm{~Hz}$, and $400 \mathrm{~Hz})$ can be clearly observed in the spectrum. The periodic impulse characteristics, in contrast, are severely submerged by the noises interference in Fig. 3(b). The energy distribution of each component in the whole signal frequency band is not obvious, and It is difficult to identify the effective frequency components reflecting fault characteristics. These experiment results demonstrate the necessity of improving the SNR of the impulse component under strong noise background.

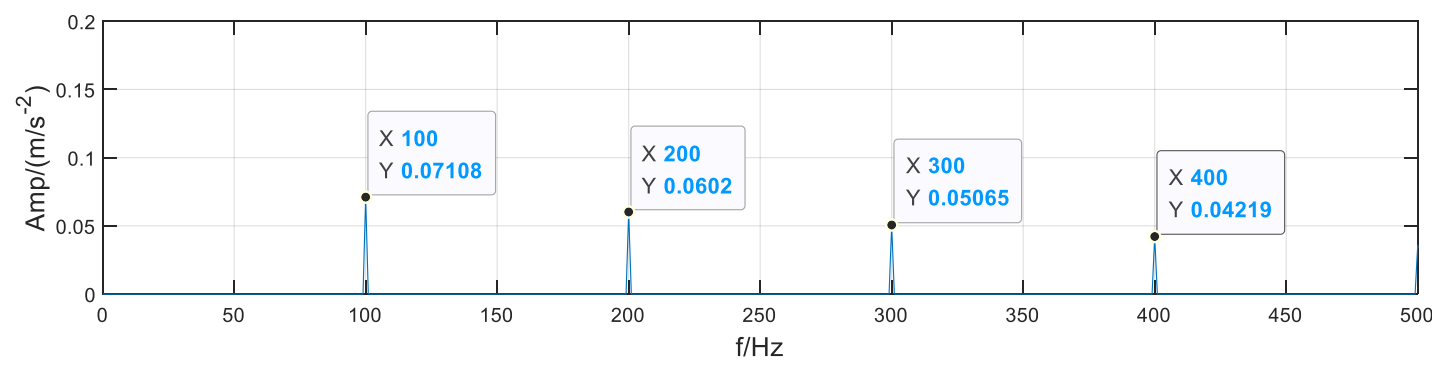

(a)

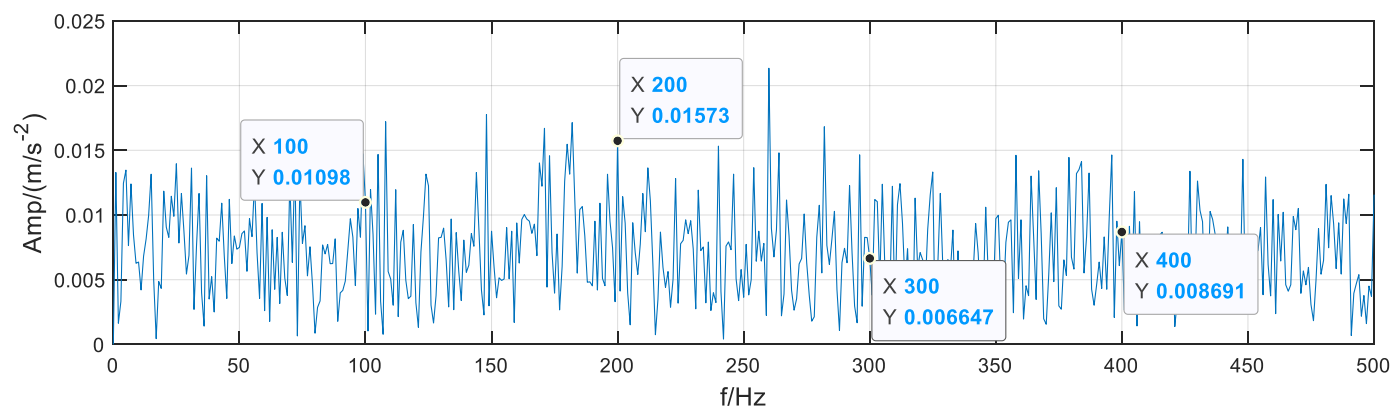

(b)

Fig.3. The envelope spectrum of shock signal (a) The periodic impulse signal (b) The impulse mixed with noise

To improve the SNR of shock characterization, Teager energy operator is introduced to enhance the impact components in the signal. In order to evaluate the advantages of the Teager energy operator method, to start with, we use existing Teager energy operator to process the pure shock signal in Fig.2, and the result is illustrated in Fig. 4. The original signal and the energy signal produce the curves 
shown in red and blue in Fig. 4 (a), respectively. It can be found that the amplitude of preprocessed energy signal has been improved. Then, the envelope spectrum of Teager energy signal is processed, and the results are shown in Fig. 4 (b). It can be observed in Fig. 4 (b) that the spectral amplitudes of fundamental frequency $(100 \mathrm{~Hz})$ and its multiplications representing fault characteristics are slightly larger than those in Fig. 3 (a). After that, the proposed CTEO is applied to analyze the raw impulse signal in Fig. 2, and the result is shown in Fig. 5 (a). By comparison, the amplitude of the energy signal preprocessed by complete Teager energy operator is improved remarkably, and the energy signal has positive and negative properties. Fig. 5 (b) shows the Hilbert envelope spectrum of CTEO signal. The spectral amplitudes of fundamental frequency and harmonic frequencies are obviously larger than those in Fig. 3 (a) and Fig. 4 (a). However, in application of available engineering, the impact signal is mixed with abundant strong background noise, as shown in Fig.2. To verify the validation of signal enhancement, the conventional TEO and the complete TEO are applied to process the mixed signal, and the results are demonstrated in Fig. 6(a) and Fig. 7(a) respectively.

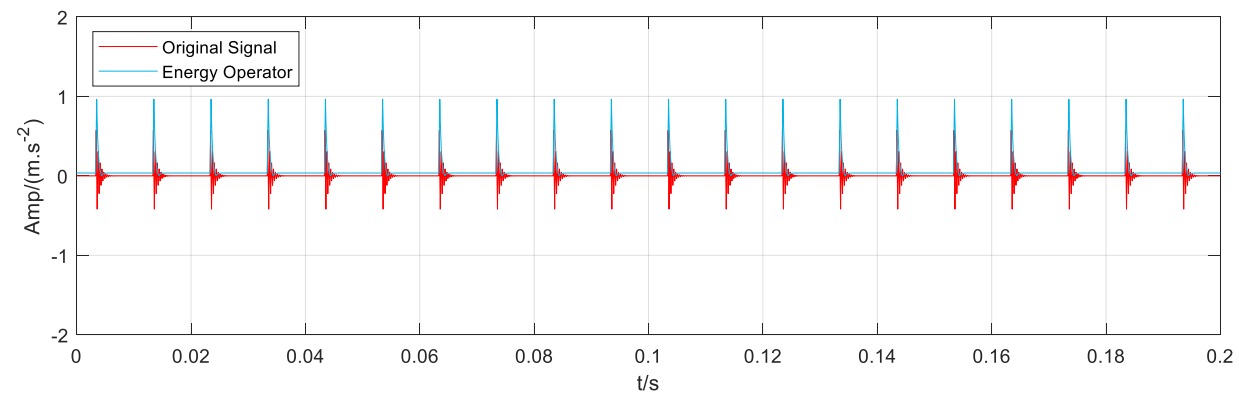

(a)

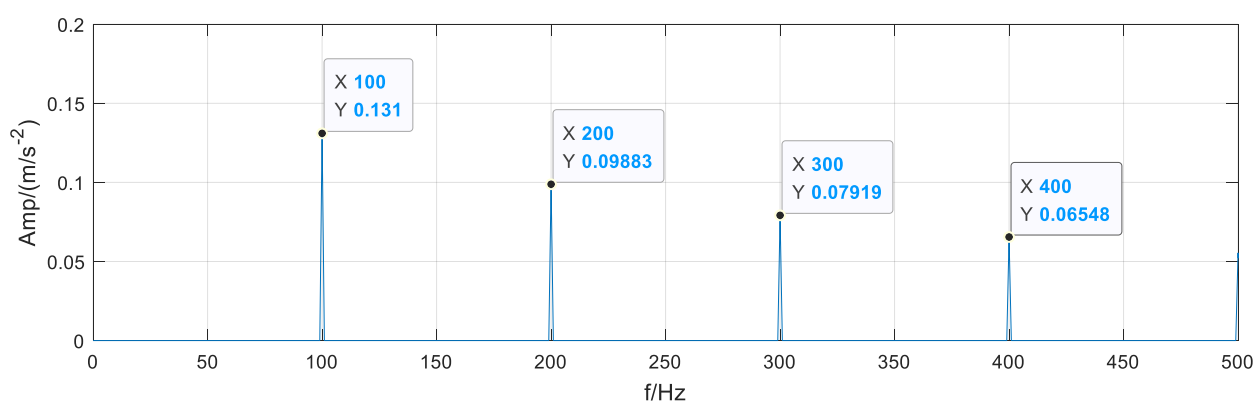

(b)

Fig.4. The original Teager modulation signal of shock signal and its envelope spectrum (a) Original Teager energy signal (b)

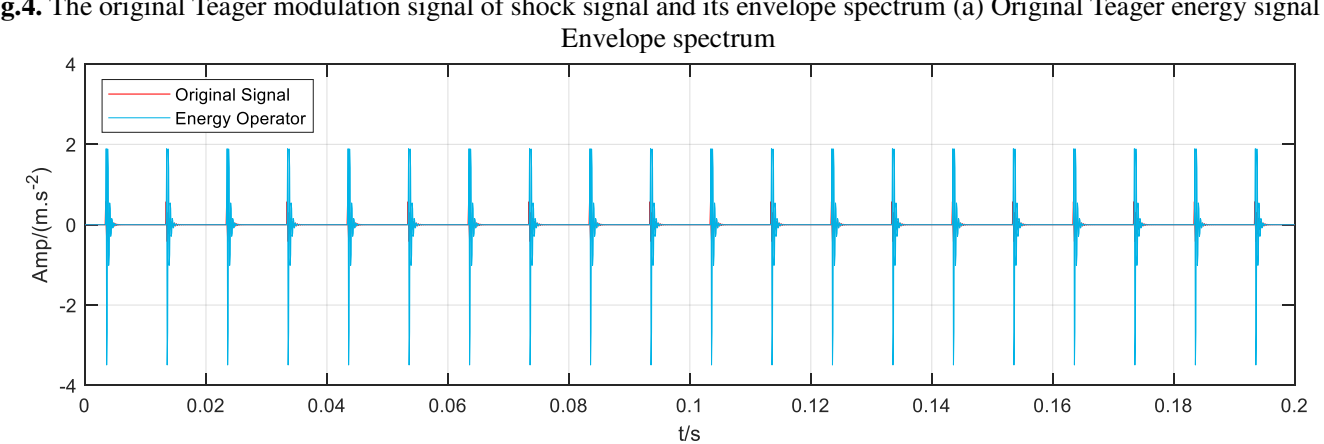

(a) 


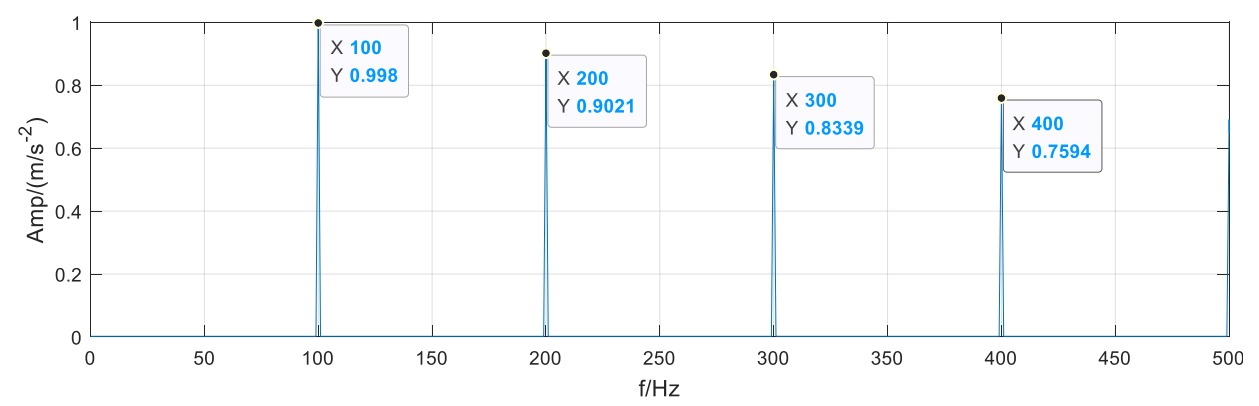

(b)

Fig.5. The proposed Teager modulation signal of shock signal and its envelope spectrum (a) The proposed Teager energy signal (b) Envelope spectrum

As shown in Fig. 6 (a), the amplitude of the energy waveform of the conventional TEO method is always positive, which is introduced in Eq. (8). From the perspective of time domain, that is, the preprocessed signal has great change with the original signal, and its frequency distribution will also change accordingly. Fig. 7 (a) describes the complete Teager energy waveform. The information is consistent with the positive and negative directions of the original signal. In other words, only the amplitude of the impact component of the original signal is enhanced without changing its directivity, and the frequency distribution is consistent with the original signal. In order to quantitatively explain the effectiveness of CTEO, the envelope spectrum of Fig. 6 (a) and Fig. 7 (a) are shown in Fig. 6 (b) and Fig. 7 (b) respectively. In Fig. 3 (b), the fault characteristic frequency $f_{i}(100 \mathrm{~Hz})$ is identified hardly and buried in the noise. Although the dual frequency component $2 f_{i}$ can be barely observed, the $3 f_{i}$ and $4 f_{i}(300 \mathrm{~Hz}$ and $400 \mathrm{~Hz})$ cannot be clearly identified. As shown in Fig. 6 (b), the energy of $f_{i}$ and its frequency harmonics are significantly improved compared with those in Fig. 3 (b). However, the amplitude of uncorrelated interference items also increase. In Fig. 7 (b), characteristic signal and its doubling frequencies have large amplitudes and distinctive features in the envelope spectrum. The SNR is greatly improved. The results show that the proposed CTEO can effectively enhance the impulse components in the signal. At the same time, the effectiveness and superiority of the proposed theory are also verified..

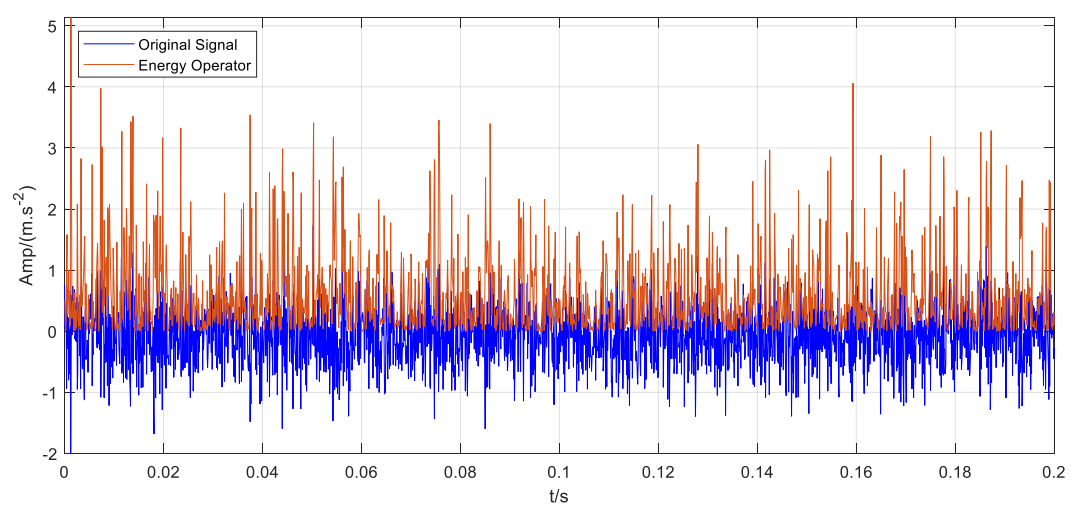

(a) 


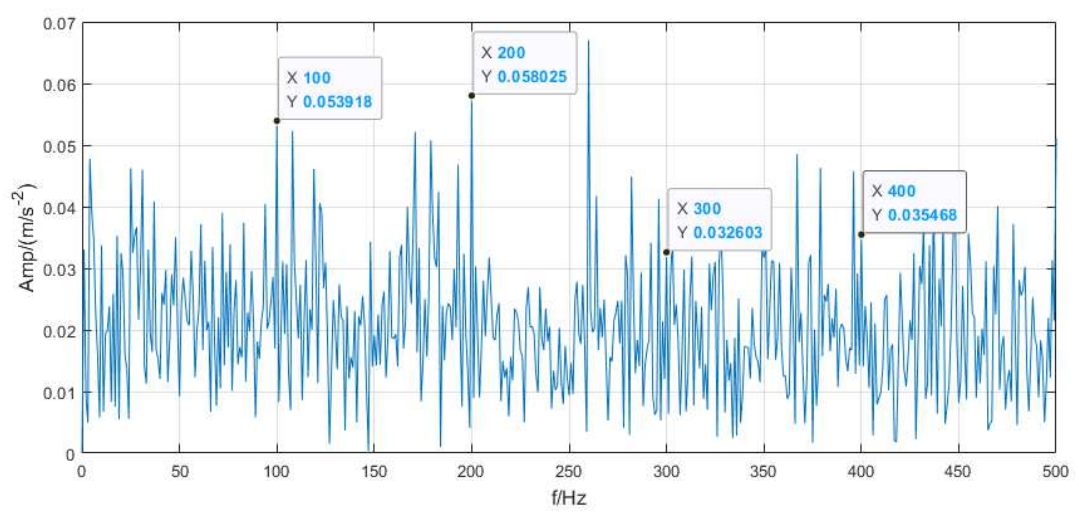

(b)

Fig.6. The conventional Teager energy signal and envelope spectrum of impulses with strong noise (a) The conventional Teager energy signal (b) Envelope spectrum

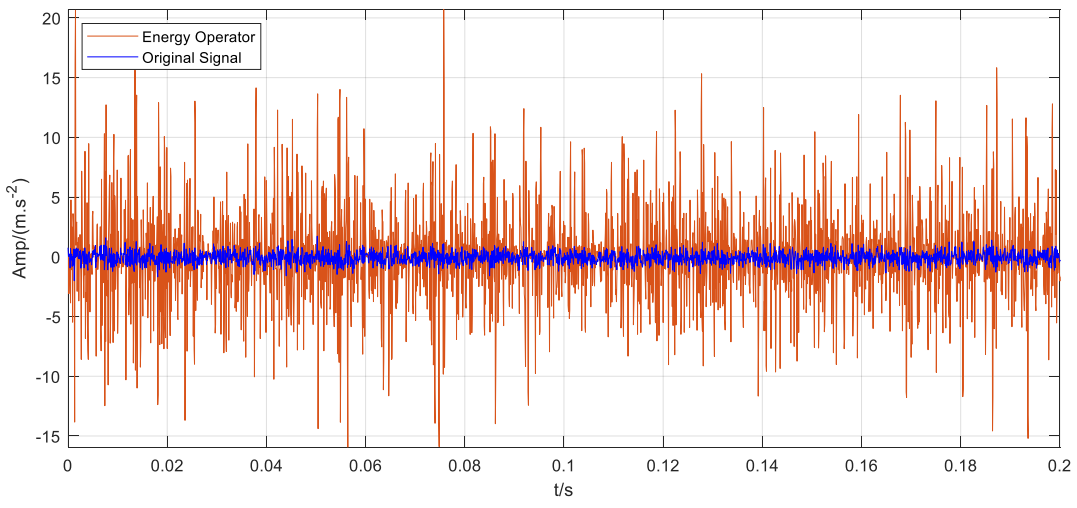

(a)

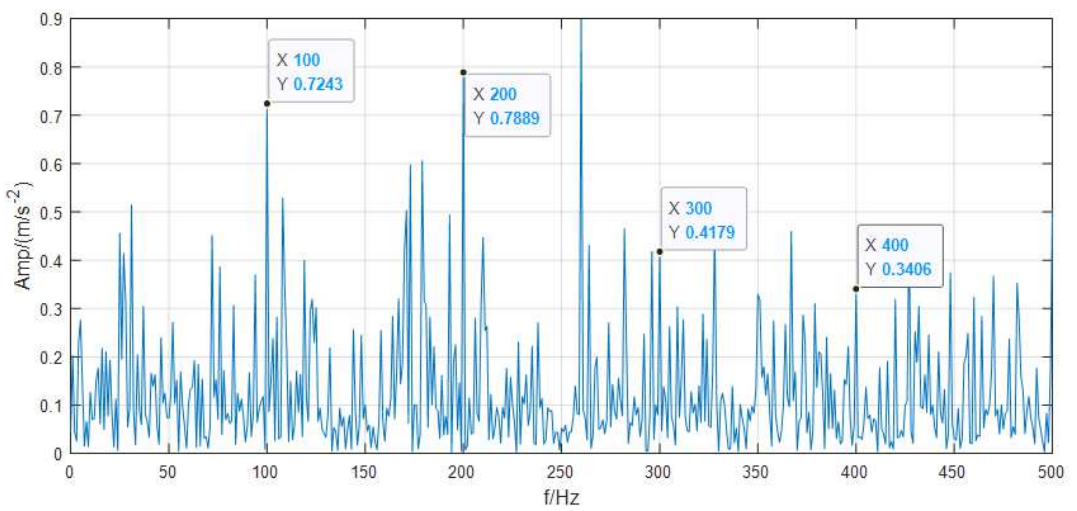

(b)

Fig.7. The complete Teager energy signal and its envelope spectrum of impulses with strong noise (a) The complete Teager energy signal (b) Envelope spectrum

The proposed CTEO method demonstrates its superiorities through these comparison results in impulse signal enhancement. It achieves much better effect for weak signal preprocessing than the conventional TEO method. To exclude the possibility of obtaining the above results due to specific simulation signal, the common platform bearing vibration signal analysis would be conducted in the next subsection.

\section{Experiment result and discussion}

In this section, the feasibility and effectiveness of the CTEO method are evaluated by analyzing the experimental data provided by Case Western Reserve University (CWRU) and the engineering signal. Furthermore, the comparisons are carried out to evaluate the advantages of the proposed 
method.

\subsection{Common experimental signal processing}

The experimental signals coming from the bearing fault test-bed of Case Western Reserve University (CWRU) are introduced to verify the validities of the proposed method [16-17]. As shown in Fig.8, the bearing fault test rig mainly consists of an induction motor, a torque transducer, a dynamometer, and several units. In the experiment, the vibration signals of rolling bearing (SKF6205) outer ring fault were collected for analysis. The signals are collected form electrical discharge machining single point damage with $0.1734 \mathrm{~mm}$ pitting diameter to simulate micro fault of bearing outer ring(including micro pulse component). The sampling frequency is $12 \mathrm{kHz}$, the sampling points are 4096, and the motor speed is $1725 \mathrm{r} / \mathrm{min}$. According to Ref. [18], the theoretical calculation of bearing outer ring fault frequency is $3.5948 * 1725 / 60=103.364 \mathrm{~Hz}$.

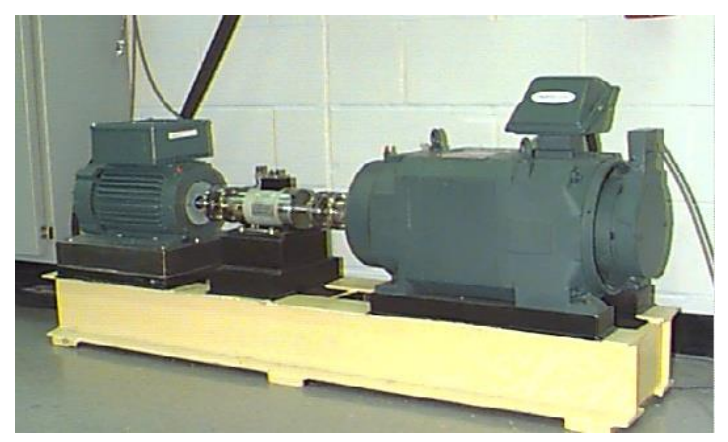

Fig.8. Rolling bearing fault test platform

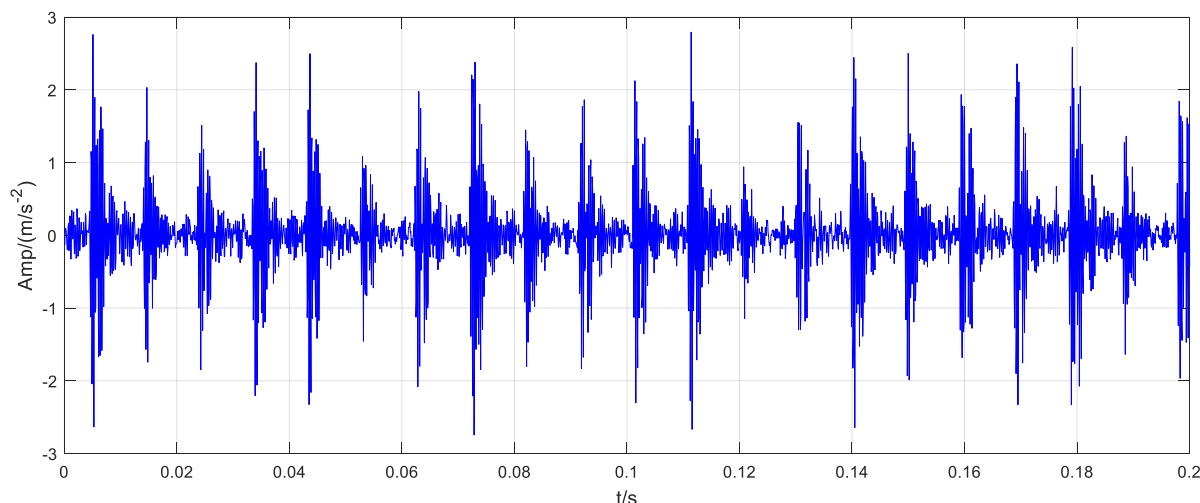

(a)

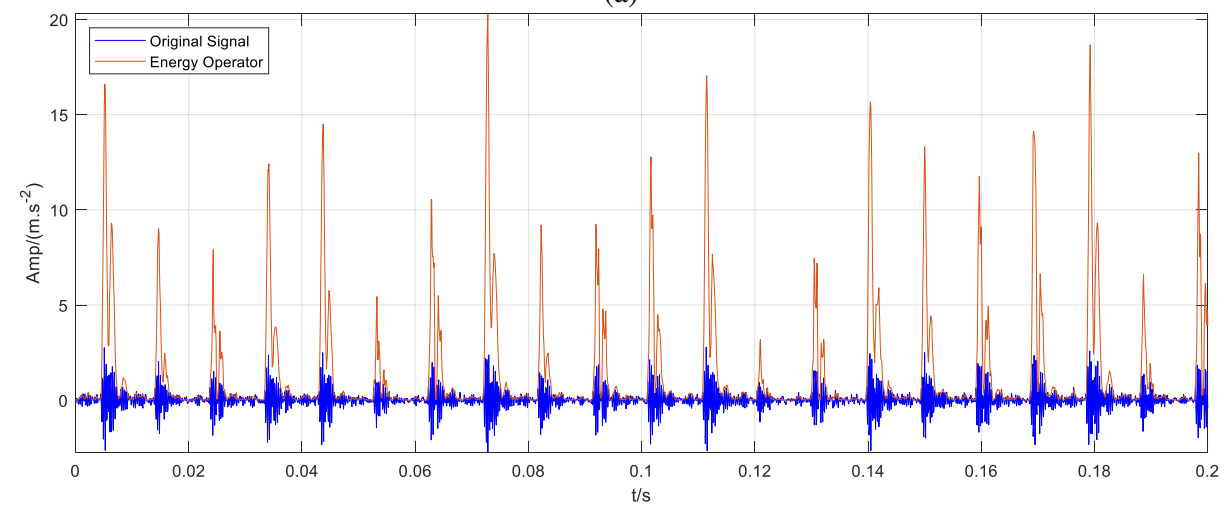

(b) 


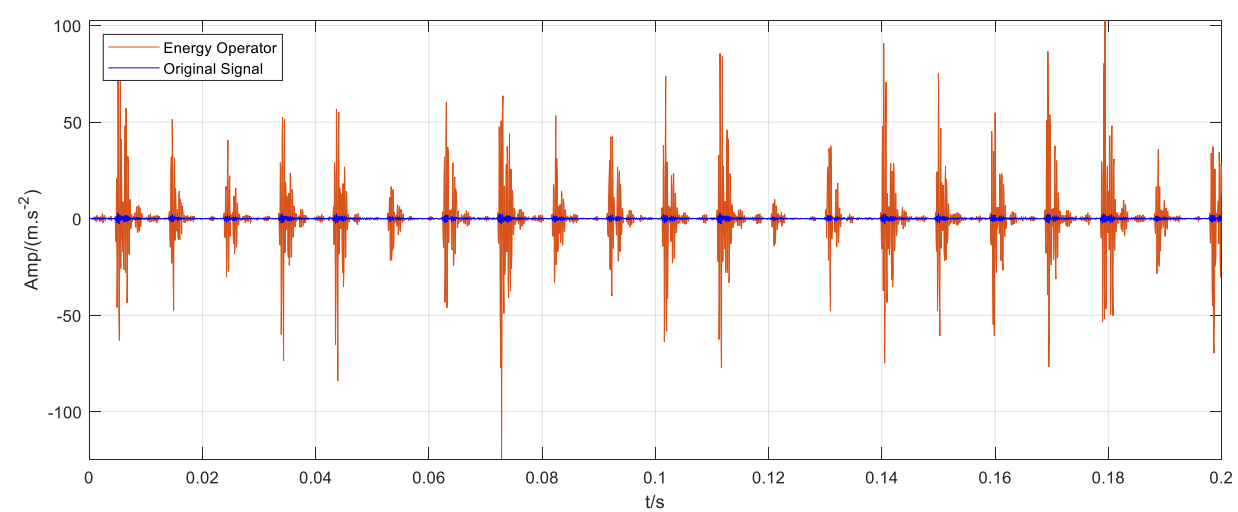

(c)

Fig.9. The bearing vibration signal and its energy signal (a) The vibration signal (b) The conventional TEO (c) The proposed TEO

The conventional TEO and the proposed TEO are used to process the raw signal of the bearing out race fault in Fig. 9 (a). The processing results are shown in Fig. 9 (b) and Fig. 9 (c), respectively. Fig. 9 (b) illustrates that the conventional TEO has good enhancement effect on the shock components in the vibration signal, but the amplitudes of preprocessing signal are all positive. As shown in Fig. 9 (c), CTEO outperforms conventional TEO in terms of amplitude, especially the directivity consistent with the original signal. In order to quantitatively describe the enhancement effect of the two Teager energy operators, the envelope spectrums of raw signal and two energy signals are presented in Fig. 10(a)-(c), respectively.

As shown in Fig. 10(a), the amplitude of rotating frequency $f_{r}(28.7 \mathrm{~Hz})$ is 0.091 . Meanwhile, the magnitudes of characteristic frequency $f_{o}(103.3 \mathrm{~Hz})$ and its frequency multiplications $\left(2 f_{o}, 3 f_{o}\right.$ and $4 f_{o}$ ) are $0.5262,0.3007,0.1967$ and 0.07718 in the envelope spectrum, respectively. Although the features can be recognized, the energy of each component is relatively small. In the spectrum of original Teager energy signal, these values in Fig. 10 (b) are 0.8143, 3.163, 1.444, 0.8226 and 0.6482 respectively. Although the identification of fault features is improved to some extent, there are still several interferences located in the low-frequency band. By introducing the envelope spectrum of complete Teager energy signal in Fig.10 (c), it can be observed that the amplitude of $f_{r}$ is 0.1651 , the amplitude of fault characteristic fundamental frequency is 5.998, and those of the second, the third, and the forth harmonics are 3.620, 1.351, and 0.692 respectively. The amplitudes of effective fault characteristic frequency components are significantly improved and the uncorrelated characteristic interference items are effectively suppressed. Compared with the original TEO methods, the proposed CTEO method has advantages in enhancing the impulse components and suppressing the unrelated noise in the original fault signals. 


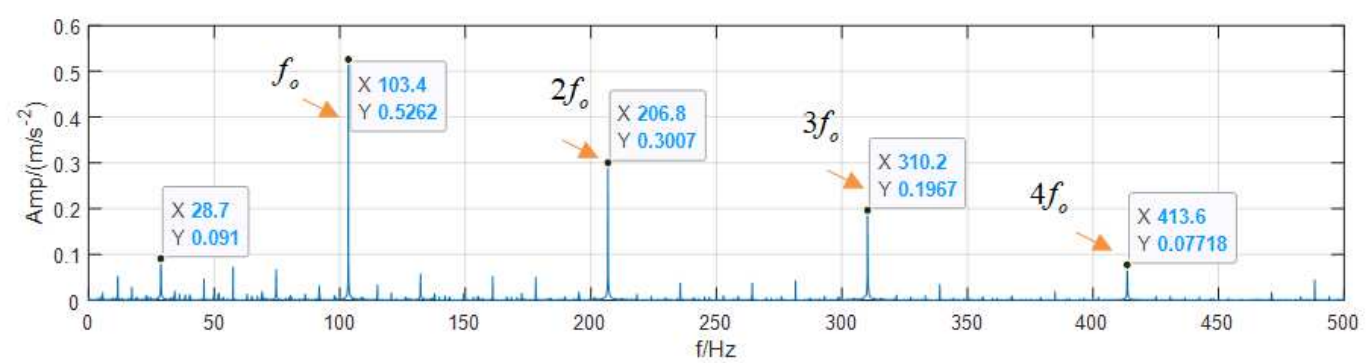

(a)

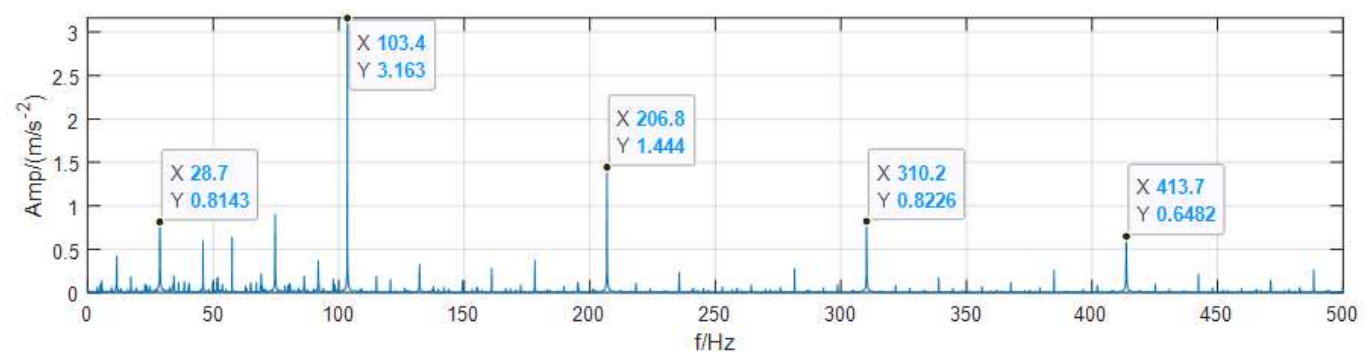

(b)

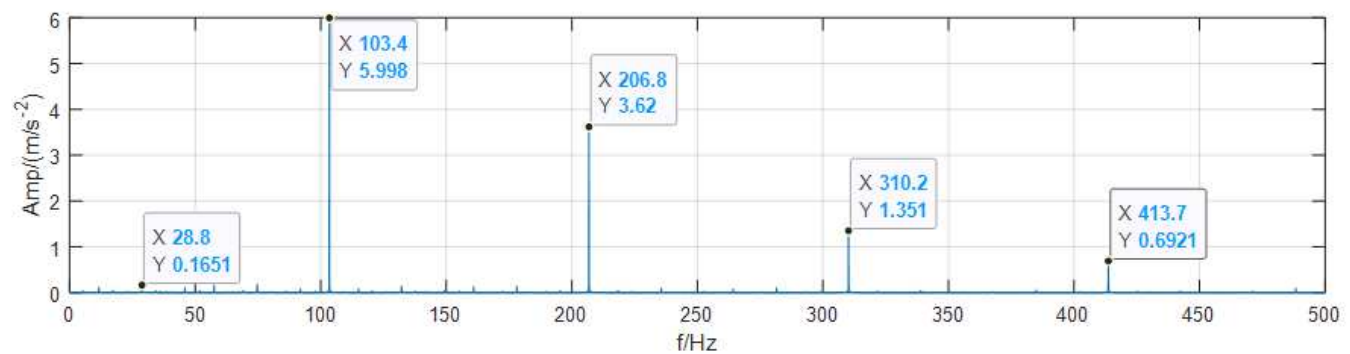

(c)

Fig.10. The envelope spectrum of experimental signal: (a)The raw signal of the out race of the bearing.(b) Conventional TEO and (c) complete TEO.

\subsection{Application}

The experimental results of the above available public data show that the method proposed in this paper is effective for the measured test set. To further verify the effectiveness of the proposed method in the actual test-bed, in this section, the weak impulse signal excitated by adjusting the bearing capacity and speed of the fault diagnosis simulator is processed. The experimental bench is shown in Fig. 11. The pitch diameter of the bearing used in the test platform is $39.5 \mathrm{~mm}$. The diameter and the number of rolling elements are $7.5 \mathrm{~mm}$ and 12 respectively .The radial load force is set as $4000 \mathrm{~N}$. Under heavy load, the rotating speed is $150 \mathrm{r} / \mathrm{min}$, and the sampling frequency is $10 \mathrm{kHz}$. According to the bearing specification and rotating speed, the fault characteristic frequency of the inner race is calculated and shown in Table 1.

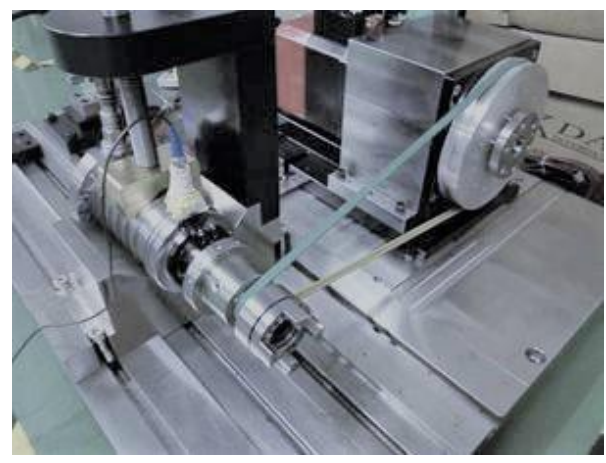

Fig.11. Experimental platform of low speed and heavy load 
Table 1 Fault characteristic frequency of the tested bearing

\begin{tabular}{|c|c|}
\hline Rotating speed(r/min) & Inner race fault characteristic frequency $\left(f_{\text {inner }} / \mathbf{H z}\right)$ \\
\hline 150 & 12.2 \\
\hline
\end{tabular}

Fig. 12(a) and Fig. 13(a) show the time-domain waveform and envelope spectrum of the raw bearing inner race fault vibration signal, respectively. As shown in Fig. 13(a), the amplitude of the rotating frequency $f_{r}(2.5 \mathrm{~Hz})$ is 0.06793 , the amplitude of the fault characteristic frequency $f_{i}(12.2 \mathrm{~Hz})$ is 0.0766 , and its dual multiplication $(22.4 \mathrm{~Hz})$ amplitude is 0.05015 . Although the fault frequency $f_{i}$ and its harmonics can be identified ( $2 f_{i}$ is low-amplitude and $3 f_{i}$ can not be clearly detected), the amplitudes of uncorrelated interference items (such as $22.6 \mathrm{~Hz}$ and $30 \mathrm{~Hz}$ ) are relatively large in the spectrum. The conventional TEO and complete TEO signals are presented in Fig. 12(b)-(c), and the corresponding Hilbert envelope spectrums are demonstrated in Fig. 13(b)-(c). As shown in Fig. 12(b)-(c), the impact components in the vibration signal have received good enhancement under the representation of the two energy operators. In Fig. 13(b), the spectral energy amplitudes of the rotating frequency and the characteristic frequency components have been greatly improved. The amplitudes of irregular interferences $(22.6 \mathrm{~Hz}$ and $30 \mathrm{~Hz})$ are relatively small, indicating that the items corresponding to those frequencies are not fault-related impulse information. In Fig.13(c), the spectrum amplitude of fault characteristic frequency $f_{i}$ is 1.2820 , and this of dual harmonic is 0.4854 . The characteristic features associated with bearing inner race fault can be identified clearly. Compared with Fig. 13(b), it demonstrates much better distinctive feature on impulse component. Especially, the shock components reflecting the fault are enhanced while the interference noises are well suppressed.

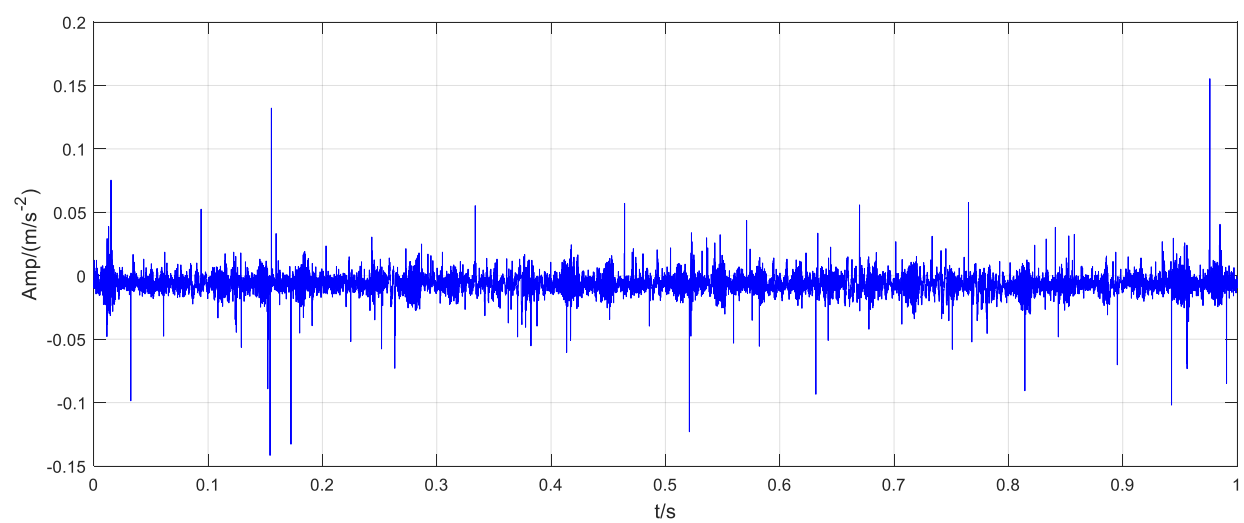

(a)

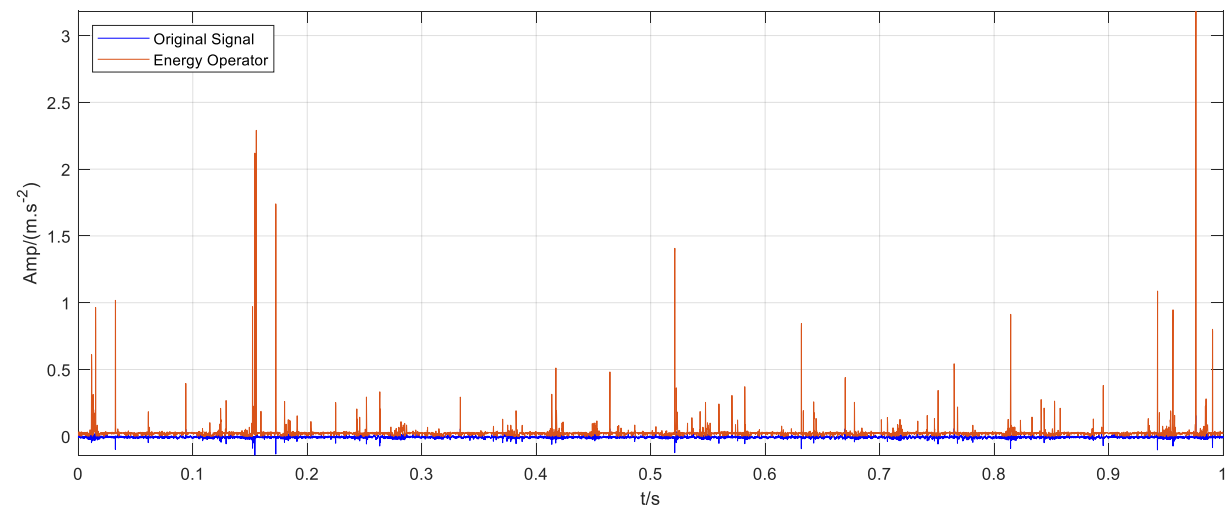

(b) 


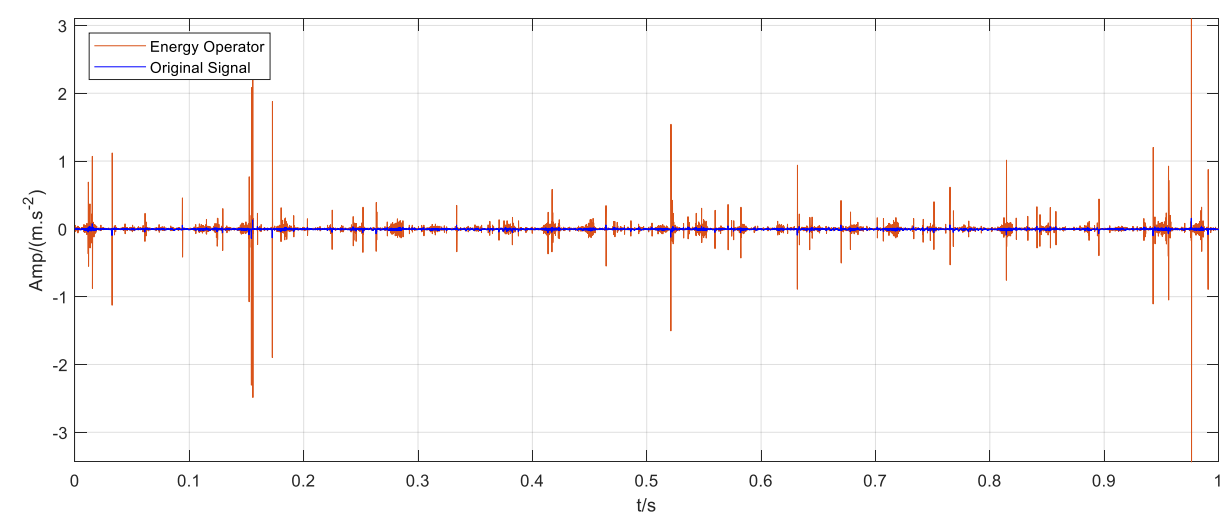

(c)

Fig.12. Raw vibration signal and its Teager energy diagram: (a) Raw signal (b) Original TEO and (c) The proposed TEO

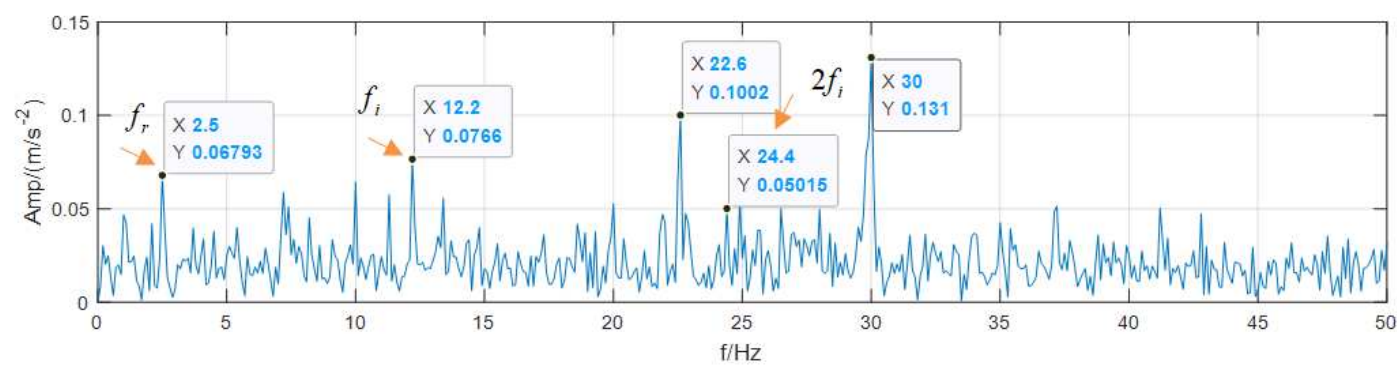

(a)

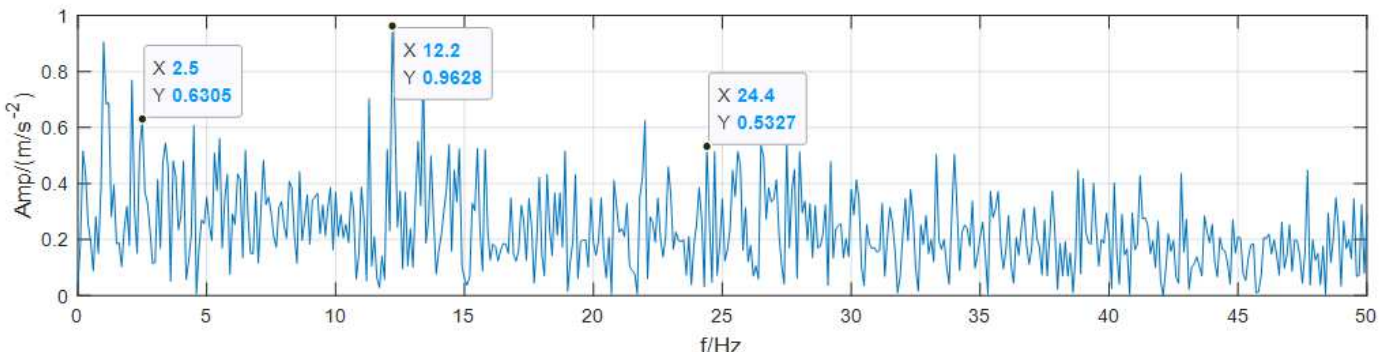

(b)

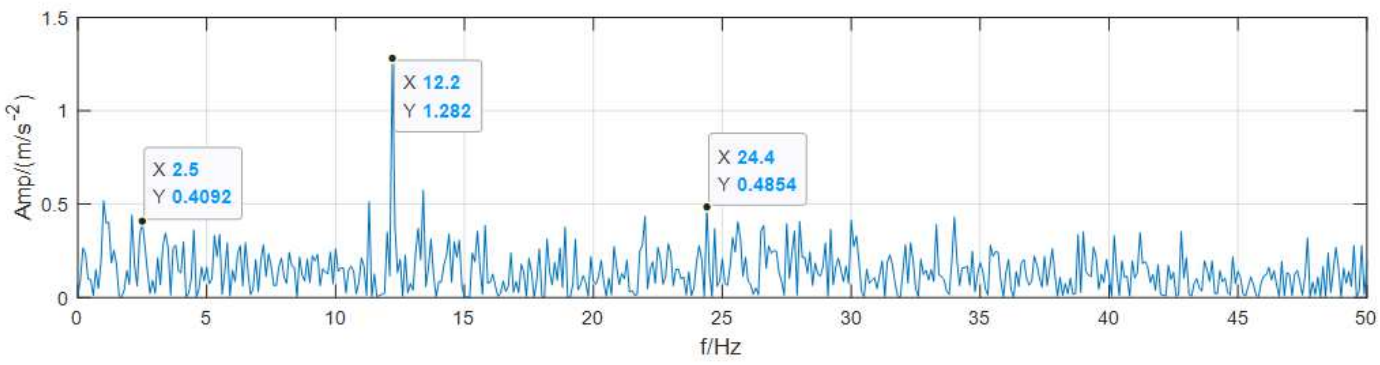

Fig.13. The envelope spectrum of experimental signal: (a) The measured signal with inner race defect. (b) The conventional TEO and (c) The proposed TEO.

In summary, this case further demonstrates the effectiveness of the proposed method to improve impulse components of the signal and restrain the other items. The signal energy representation is the accurate value of the squared product of its instantaneous amplitude $(A)$ and the instantaneous frequency $(\Omega)$. Complementarily, the method maintains the positive and negative distribution of the shock signal $\left(x_{n}[\cdot]\right)$, effectively enhances the fundamental frequency energy of the shock signal, and provides a new approach to improving the signal-to-noise ratio of the weak shock signal.

\section{Conclusion}

In this paper, an impulses enhancement method, named complete Teager energy operator is proposed based on the traditional Teager energy operator. The method focuses on weak feature enhancement of 
transient signal in low amplitude. The validity and stability of the proposed method are evaluated by simulation signal analysis, common fault signal testing and fault signal of low speed machinery with heavy load. The proposed method demonstrated better performance than the traditional Teager energy operator in enhancing the efficient impulse components of the signal.

\section{Abbreviations}

TEO: Teager energy operator; CTEO: Complete Teager energy operator; SNR: Signal-to-noise ratio

\section{Availability of data and material}

The datasets used and/or analyzed during the current study are available from the corresponding author on reasonable request.

\section{Competing interests}

The authors declared no potential conflicts of interest with respect to the research.

\section{Funding}

This work was supported in part by the National Natural Science Foundation of China under Grant 51975433 and Grant 51975430, in part by the Natural Science Foundation of Hubei Province under Grant 2019CFB133 and Grant B2020162.

\section{Authors' contributions}

XD and HPL designed research, performed research, analyzed data, and wrote the paper.

\section{Authors' information}

HPL has a Ph.D. in Control Engineering; has a background in digital signal processing and machine learning; XD has a Master's degree in Structure Engineering and specialize in construction vibration signal detecting.

\section{Acknowledgement}

The authors would like to thank the members of the Engineering Research Center for Metallurgical Automation and Measurement Technology of Ministry of Education for their support and contributions to this research. 


\section{Reference}

[1] Debadatta AG, Ranjan KM. Microgrid differential protection scheme using downsampling empirical mode decomposition and Teager energy operator. Electric Power Systems Research 2019; 173(2019): 173-182.

[2] Han T, Liu QN, Zhang L, Tan ACC. Fault feature extraction of low speed roller bearing based on Teager energy operator and CEEMD. Measurement 2019; 138(2019): 400-408.

[3] Li LL, Cui Y H, Chen RL, Chen, LP, Wang, LH. Research on Rolling Bearing Fault Feature Extraction Based on Singular Value Decomposition considering the Singular Component Accumulative Effect and Teager Energy Operator. Shock and Vibration, Volume 2019, Article ID 3742512, 14 pages.

[4] Yan BK, Wang B, Zhou FX, Li WG. Sparse decomposition method based on time-frequency spectrum segmentation for fault signals in rotating machinery, ISA Transactions 2018; 83(2018): 142-153.

[5] Rahman MM, Uddin MN. Online Unbalanced Rotor Fault Detection of an IM Drive Based on Both Time and Frequency Domain Analyses. IEEE Trans Ind App 2017; 53(4): 4087-4096.

[6] Kedadouche M, Liu ZH, Vu VH. A new approach based on OMA-empirical wavelet transforms for bearing fault diagnosis. Measurement 2016; 90: 292-308.

[7] Huang NE, Shen Z, Long S. The empirical mode decomposition and the Hilbert spectrum for nonlinear and non-stationary time series analysis [M]. London: The Royal Society, 1998.

[8] Wang L, Cai GG, Wang J. Jiang XX, Zhu ZK. Dual-Enhanced Sparse Decomposition for Wind Turbine Gearbox Fault Diagnosis [J]. IEEE Transactions on Instrumentation and Measurement, 2019.68(2): 450-461.

[9] Xu B, Zhou FX, Li HP, et al. Early fault feature extraction of bearings based on Teager energy operator and optimal VMD, ISA Transactions, 2019, 86(2019): 249-265.

[10] Gadanayak DA, Mallick RK. Microgrid differential protection scheme using downsampling empirical mode decomposition and Teager energy operator. Electric Power Systems Research, 2019, 173 (2019): 173-182.

[11] Paul R, Sengupt A. Discrete wavelet packet transform based controller for liquid level system and its performance analysis. Measurement, 2017, 97(2017): 226-233.

[12] Gu R, Chen J, Hong RJ, et al. Incipient fault diagnosis of rolling bearings based on adaptive variational mode decomposition and Teager energy operator. Measurement 2020;149(2020): 106941.

[13] Maragos P, Kaiser JF, Quatieri TF. On amplitude and frequency demodulation using energy operators, IEEE Trans. Signal Process. 41 (4) (1993) 1532-1550.

[14] Kaiser JF. On a simple algorithm to calculate the 'energy' of a signal, in: Int. Conf. on Acoustics, Speech, and Signal Process 1990; pp. 381-384.

[15] Yan BK, Wang B, Zhou FX. Sparse feature extraction for fault diagnosis of rotating machinery based on sparse decomposition combined multiresolution generalized S transform. Journal of Low Frequency Noise, Vibration and Active Control2019; 0(0): 1-16.

[16] Loparo K. "Bearings vibration data set," Case Western Reserve University, < http://csegroups.case.edu/bearingdatacenter/pages/download-data-file $>$.

[17] Smith WA, Randall RB. Rolling element bearing diagnostics using the Case Western Reserve University data: A benchmark study. Mech. Syst.Signal Process 2015; 64:100-31.

[18] Gong X, Qiao W. "Bearing fault diagnosis for direct-drive wind turbines via current-demodulated signals," IEEE Trans. Ind. Electron. 2013; 60( 8): 3419-3428. 